\title{
The Effect of Inoculum Size on Inhibition Zones in Agar Media Using Staphylococci and Streptomycin
}

\author{
BY K. E. COOPER, A. H. LINTON AND S. N. SEHGAL \\ Department of Bacteriology, University of Bristol
}

\begin{abstract}
SUMMARY : Investigations on the size of inhibition zones in agar tube cultures of Staphylococcus aureus (Mayo) due to diffusion of streptomycin have confirmed the formula previously suggested. No significant deviation from expectations has been found over a temperature range of $27-40^{\circ}$, nor with variations of inoculum size from $2 \times 10^{3}$ to $5 \times 10^{8}$ organisms $/ \mathrm{ml}$. The theoretical basis of the formula has been further elucidated and the meaning of critical population, critical concentration and critical time clarified. These constants are determined by the necessity of absorbing a critical amount of antibiotic on the organisms to inhibit growth within the zone. To maintain this absorption a minimum inhibitory concentration must remain in the surrounding medium.
\end{abstract}

Cooper \& Woodman (1946) proposed, on theoretical grounds, a formula for the size of antibiotic inhibition zones in agar media and Cooper (1955) extended this formula by elucidating the nature of the factors which control the time during the growth of the organism at which the size of the zone is determined. This critical time $T_{0}$ is given by:

$$
T_{0}=L+G \log _{2}\left(N^{\prime} / N_{0}\right)=X^{2} / 4 D \log _{e}\left(m_{0} / m^{\prime}\right),
$$

where $L=$ lag period in viable count; $G=$ generation time; $N_{0}=$ number of organisms in inoculum/unit volume final medium; $N^{\prime}=$ critical population reached at time $T_{0}$ after inoculation, per unit volume of agar; $X=$ depth of inhibition zones; $\boldsymbol{D}=$ diffusion coefficient of antibiotic; $m_{0}=$ concentration of antibiotic in contact with the agar surface in the tube; $m^{\prime}=$ critical concentration at distance $X$ from surface at time $T_{0}$.

The conditions under which this theoretical formula is expected to be true are necessarily those under which the initial assumptions are most nearly approached. The most important are: an antibiotic which diffuses freely through a non-interfering medium from a reservoir of constant concentration, and an inoculation of a determined number of metabolizing organisms which, after a constant lag period, multiply at a constant rate. Constancy of temperature and of media composition are also important. Many workers have examined the validity of the formula of Cooper \& Woodman (1946) which connects the zone size with the diffusion laws and critical time; these include Vesterdal (1947), Masuyama (1948), Herdan (1949), Linton (1949), Sykes, Lewis \& Goshawk (1951), Miyamura (1953), Schonfeld (1953), Ingram, Foxell \& Armitage (1953), Schlesinger (1954) and Gillissen (1954). Others have, in addition, contributed to our knowledge of the relationship between critical time and the growth of the organism. These are Mitchison \& Spicer (1949), Cooper \& Gillespie (1952), Cooper \& Linton (1952), Cooper (1952), Humphrey 
\& Lightbown (1952), Linton (1954, 1955), Cooper (1955). In the first paper of Cooper \& Woodman (1946) it was shown that zones could be abolished by pre-incubating cultures before beginning the diffusion of the antibiotic. When growth proceeds for $h \mathrm{hr}$. after time 0 , before the antibiotic is added, the time of diffusion is given by

$$
T_{0}-h=X^{2} / 4 D \log _{e}\left(m_{0} / m^{\prime}\right) .
$$

When $h=0$ (the usual condition in assays), then the time of diffusion becomes the same as the time of growth, $T_{0}$. When $h=T_{0}$, then growth has reached the critical population $N^{\prime}$ before diffusion starts and no zone of inhibition is produced. When the size of the inoculum $\left(N_{0}\right)$ is increased, then the time $T_{0}$ required to reach the critical population should be diminished. A critical survey of the influence of inoculum size upon the size of inhibition zones at different temperatures has hitherto not been published; this is the object of the present paper. We have investigated this for the action of streptomycin on sensitive and resistant strains of Staphylococcus aureus in a constant medium.

\section{METHODS}

Organisms. (i) The 'Mayo' strain of Staphylococcus aureus was used as a streptomycin-sensitive organism (our collection reference No. 6473). This strain was kept freeze-dried and retained a constant $m^{\prime}$ value of $2.5 \mu \mathrm{g}$. streptomycin/ml. Earlier experiments with this strain before freeze-dried stocks were kept showed slightly greater sensitivities with an $m^{\prime}$ value as low as $1 \cdot 8 \mu \mathrm{g} . / \mathrm{ml}$. (see Table 1).

(ii) A resistant mutant, with the same growth rate as the 'Mayo' staphylococcus, was derived from this strain. This also was freeze-dried and retained a constant $m^{\prime}$ value of $\mathbf{7 \cdot 0 8} \mu \mathrm{g}$. streptomycin/ml. (our collection reference No. 6474).

For a series of experiments both strains were maintained on nutrient agar slopes, subcultured at weekly intervals and kept at room temperature. A fresh culture from a freeze-dried ampoule was used when the $m^{\prime}$ value was found to alter as a result of repeated subculture.

Standard broth was used in the preparation of the inocula and for the determination of generation time and lag periods. It was prepared with the following ingredients: Bacto-peptone, $10 \mathrm{~g}$.; $\mathrm{NaCl}, 5 \mathrm{~g}$; beef heart infusion made with glass-distilled water, $500 \mathrm{ml}$.; and the volume made up to $1000 \mathrm{ml}$. with glass-distilled water. After adjusting to $\mathrm{pH} \mathbf{7 \cdot 8}$, it was dispensed in screw-cap bottles (100 ml. quantities) sterilized by autoclaving and stored in the dark.

Standard agar. For diffusion experiments, the standard broth was gelled with $1 \%(\mathrm{w} / \mathrm{v})$ Bacto-agar.

Antibiotic. Streptomycin calcium chloride (Glaxo) was diluted in sterile glass-distilled water to give a final concentration of $200 \mathrm{mg}$. streptomycin $/ \mathrm{ml}$. This was stored at $4^{\circ}$ in screw-cap bottles and replaced at fortnightly intervals. Dilutions were made from this stock solution in sterile glass-distilled water 
immediately before each experiment was set up, to contain 10, 100 and $1000 \mu \mathrm{g}$. streptomycin $/ \mathrm{ml}$.

Preparation of inocula for diffusion experiments. The organism to be tested was inoculated into $10 \mathrm{ml}$. standard broth in a plugged tube, and incubated for $20 \mathrm{hr}$. at $37^{\circ}$. Immediately before beginning the experiment, this culture was diluted in sterile glass-distilled water warmed to $37^{\circ}$, to give a series of dilutions. These were planned so that when $0.5 \mathrm{ml}$. was added to $9.5 \mathrm{ml}$. of molten standard agar, held at $45^{\circ}$ in a water bath, the final number of viable organisms approximated to the required counts. The viable count of the $20 \mathrm{hr}$. broth culture was determined by the technique of Miles \& Misra (1938) for each series of experiments and the size of each inoculum was calculated from this.

\section{Diffusion experiments}

Procedure. The assay method of Mitchison \& Spicer (1949) was used, with certain modifications described by Cooper \& Gillespie (1952). By this method, molten standard agar, after inoculation, was immediately pipetted into sterile glass tubes $(3 \times 75 \mathrm{~mm}$.), to a depth of $c .40 \mathrm{~mm}$. Mitchison \& Spicer showed that variation of degree of anaerobiosis by alteration of depth of column was without influence on the size of the zones in the case of streptomycin. The tubes were placed in racks in the upright position and the agar allowed to set by standing at room temperature for $10 \mathrm{~min}$. The racks of tubes were then incubated in water baths regulated at the required temperatures. The time at which incubation was begun was regarded as time 0 . Three concentrations of streptomycin $(10,100,1000 \mu \mathrm{g} . / \mathrm{ml}$.) were pipetted on to the agar surface of the first of three series of tubes at time 0 and, thereafter, at convenient periods of pre-incubation, e.g. 1, 2, 3, etc. hr. The depths of the zones of inhibition, produced by diffusion of the streptomycin, were read after $20 \mathrm{hr}$. incubation. The square of the values $\left(X^{2}\right)$ thus obtained, for each concentration of streptomycin and for each inoculum size, were plotted in various ways in order to determine the constants $T_{0}, m^{\prime}$ and $D$ in the formula (Cooper, 1955).

Determination of $T_{0}$. When the values of $X^{2}$ were plotted against the time of pre-incubation $(h)$ it was found that, independent of the concentration of streptomycin, straight lines which connected the experimental points intersected the $h$ axis at time $T_{0} \mathrm{hr}$. This value was determined for each inoculum size $\left(N_{0}\right)$ investigated, over a range from $2 \times 10^{3}$ to $5 \times 10^{8}$ viable organisms $/ \mathrm{ml}$.

Determination of $m^{\prime}$. Plotting the values of $X^{2}$ against the logarithm of the concentrations of streptomycin used in the experiments $(10,100,1000 \mu \mathrm{g} . / \mathrm{ml}$.) resulted in straight-line graphs being obtained for each value of $h$ and these intersected on the concentration axis at $m^{\prime}$.

Determinations of $D$. This constant was calculated by substituting experimental values of $X^{2}, T_{0}, m^{0}$ and $m^{\prime}$, obtained at $35^{\circ}$, in the formula

$$
T_{0}=X^{2} / 4 D \log _{\boldsymbol{e}}\left(m^{0} / m^{\prime}\right) \text {. }
$$

Inocula of different sizes were used over the whole range possible and the mean value of a large number of determinations was adopted. Values of $\boldsymbol{D}$ for other 
temperatures were obtained theoretically, by correcting the value at $35^{\circ}$ for the change in the viscosity of water with temperature, by means of the formula:

$$
D_{1} / D_{2}=T_{1} \eta_{2} / T_{2} \eta_{1}
$$

where $D_{1}$ and $D_{2}$ are the diffusion coefficients at absolute temperatures $T_{1}$ and $T_{2}$ and $\eta_{1}$ and $\eta_{2}$ are the viscosities of water at these temperatures (see Cooper \& Woodman, 1946).

The effect of inoculum size on inhibition zones. In order clearly to demonstrate this, the experimental values of $X^{2}$ for $h=0$ have been plotted against the logarithm of the inoculum size, $\log _{2} \mathrm{~N}_{0}$ (Figs. $1 b, 2 b$ and $3 b$ ). This was done with the sensitive staphylococcus for each concentration of streptomycin at one temperature (Fig. $1 b$ ) and similarly with the resistant mutant (Fig. 3b). Results are given for one concentration of streptomycin at different temperatures (Fig. $2 b$ ). In addition the $T_{0}$ values determined under the same conditions have been plotted against the logarithm of the inoculum size (Figs. 1a, $2 a$ and $3 a$ ).

\section{Determination of generation time and lag periods}

Broth experiments. The generation time and lag periods were determined in standard broth for both the sensitive and resistant staphylococci and at each temperature at which the diffusion experiments were done. These constants were determined by using inocula of approximately $1.0 \times 10^{6}$ viable organisms/ $\mathrm{ml}$., and under conditions as similar as possible to those of the diffusion experiments. This included diluting a $20 \mathrm{hr}$. broth culture in sterile glassdistilled water (1/50), adding $0.5 \mathrm{ml}$. of this to $9.5 \mathrm{ml}$. of standard broth warmed to $45^{\circ}$, cooling at room temperature for $10 \mathrm{~min}$. and then beginning incubation in regulated water baths. The generation time and lag periods were determined by viable counts on samples removed at regular intervals.

Agar experiments. An attempt was made to determine the generation time in solid medium. Inoculated tubes of standard agar were prepared as for diffusion experiments and incubated without streptomycin. At intervals of time, tubes were taken from the water bath and their sealed ends cut off with a glass file to facilitate removal of the agar columns. These were fixed in formalin for $12 \mathrm{hr}$. and washed in distilled water for a further $4 \mathrm{hr}$. They were then floated on to glass slides, dried at $37^{\circ}$ in the incubator and stained by Gram's method. The number of organisms which had developed in each colony were counted by direct microscopy. Though the method was not very accurate, reasonable agreement was obtained with the determinations in broth.

\section{RESULTS}

Many experiments were undertaken with a view to determining quantitatively the effect of heavy and light inocula on the size of inhibition zones formed by diffusion of inhibitory concentrations of streptomycin. Only some examples of the results can be given here and are illustrated by Figs. 1-3.

Figures $1 a$ and $1 b$ illustrate the results obtained at $35^{\circ}$, with the sensitive 'Mayo' staphylococcus. For each inoculum size used, $m^{\prime}$ was determined, and 
the value of $1 \cdot 8 \mu \mathrm{g}$. streptomicin $/ \mathrm{ml}$. was found to be constant throughout the series. The extrapolated values of $\boldsymbol{T}_{0}$ were separately determined for each inoculum size and became smaller as the inoculum size was increased. Each value of $T_{0}$ was obtained from experimental readings of $X^{2}$ for all periods of preincubation $(h)$, from nine to twenty-seven readings being used to deter-

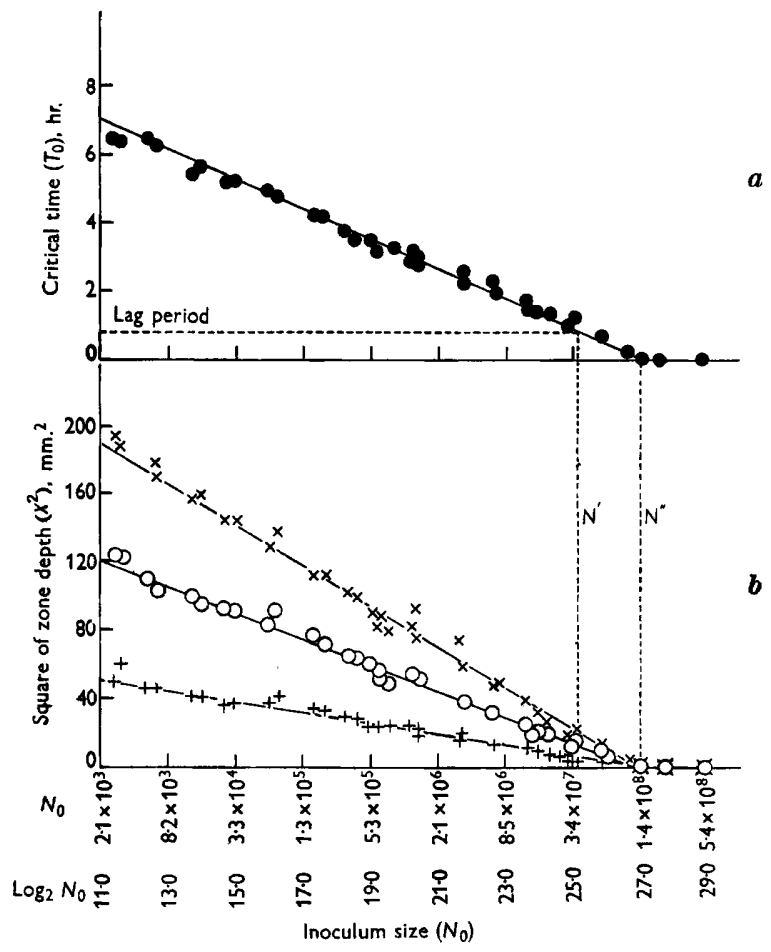

Fig. 1. The effect of inoculum size on critical time and the depth of inhibition zones produced by the action of streptomycin on staphylococcus (Mayo 6473) at 35 ${ }^{\circ}$ (a) Critical time $\left(T_{0}\right)$ plotted against inoculum size $\left(\log _{2} N_{0}\right)$. The points are the extrapolated values of $h$ obtained from graphs plotting zone size $\left(X^{2}\right)$ against hours of preincubation $(h)$, since when $X^{2}=0$, then $h=T_{0}$. Three lines for different concentrations of antibiotic $\left(m_{0}=10\right.$, $100,1000 \mu \mathrm{g} . / \mathrm{ml}$.) were extrapolated and the average value used for each size of inoculum tested $(O)$. (b) Zone size $\left(X^{2}\right)$ determined in triplicate experiments, for $m_{0}$ values of $10 \mu \mathrm{g} . / \mathrm{ml}$. ( + ), $100 \mu \mathrm{g} . / \mathrm{ml}$. (O), $1000 \mu \mathrm{g} . / \mathrm{ml}$. ( $\times$ ), when $h=0$ plotted against inoculum size. $N^{\prime}$ is the critical population, which all inocula less than $N^{\prime}$ achieve at time $T_{0}$ when the zone edge is formed. $N^{\prime \prime}$ is that size of inoculum $\left(N_{0}=N^{\prime \prime}\right)$ which reduces $T_{0}$ and $X^{2}$ to nil. For theoretical constants used to calculate the graphs, see Table 1.

mine each point. The values of $T_{0}$ have been plotted against the logarithm of the inoculum size, $\log _{2} N_{0}$ (Fig. $1 a$ ) and the best straight line through these points intersects the inoculum size axis at $\log _{2} N_{0}=27 \cdot 0 \quad\left(N=135.5 \times 10^{6}\right.$ organisms $/ \mathrm{ml}$.). Inocula of greater size than this are too great to allow the formation of inhibition zones, and, therefore, $T_{0}=0$ in these cases.

In Fig. $1 b$, the values of $X^{2}$, obtained with each of three concentrations of streptomycin $(10,100,1000 \mu \mathrm{g} . / \mathrm{ml}$.), are plotted against the logarithm of the 
inoculum size. It is seen that the size of the inhibition zones is decreased to zero when the inoculum size is given by $\log _{2} N_{0}=27 \cdot 0$. This value of $N_{0}$, which is independent of the concentration of the antibiotic used, we have represented by $N^{\prime \prime}$.

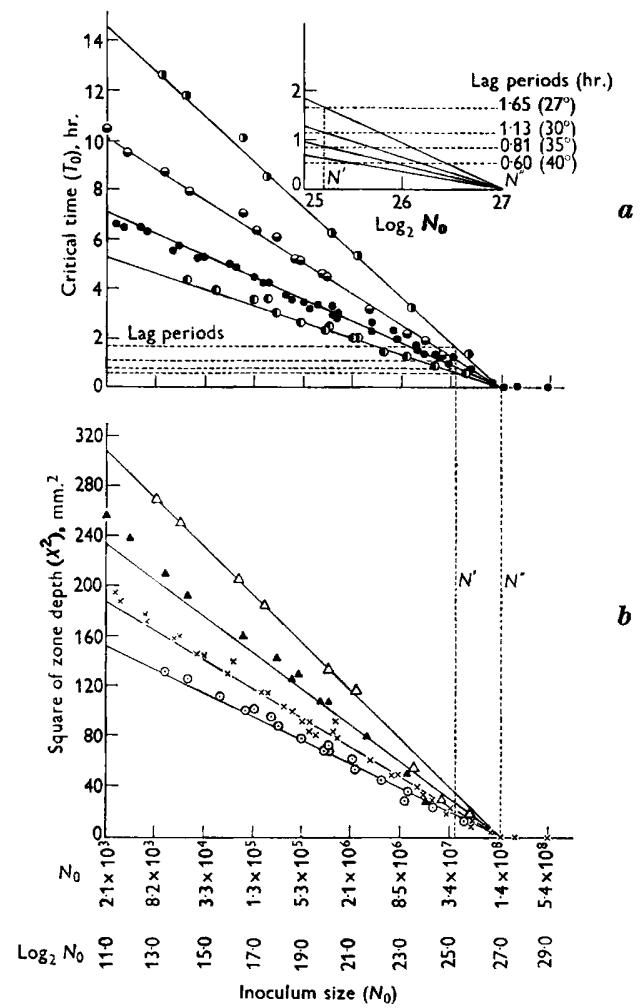

Fig. 2. The effect of inoculum size on critical time and the depth of inhibition zones produced by the action of streptomycin on staphylococcus (Mayo 6473) at different temperatures. (a) As in graph $1 a$. When $N_{0}=N^{\prime}$ then $T_{0}=L$, and the inset graph shows the theoretical values of $L$ at these temperatures. $27^{\circ}, 0 ; 30^{\circ}, \ominus ; 35^{\circ}, 0 ; 40^{\circ}, O$. (b) As in graph $1 \mathrm{~b}$, but values of $X^{2}$ are plotted for one value of $m_{0}(=1000 \mu \mathrm{g} . / \mathrm{ml}$.) only, for each temperature. $27^{\circ}, \triangle ; 30^{\circ}, \Delta ; 3^{\circ}, \times ; 40^{\circ}, \odot$.

The calculation of regression lines through the points for $\log _{2} N_{0}$ plotted against $T_{0}$, gives a value of $N^{\prime \prime}$ which $\pm 2 \sigma$ ( $\sigma=$ standard deviation) overlaps $N^{\prime \prime}$ values calculated from the regression lines of the $X^{2}$ values for each concentration. $N^{\prime \prime}$ is thus independent of the concentration of the antibiotic and within the limits of experimental error lies on the extrapolation of the best straight lines determined for any workable range of sizes of inocula. The value $\log _{2} N^{\prime \prime}=\mathbf{2 7 \cdot 0}$ has been chosen as the most probable value for all our experiments, including those at temperatures other than $35^{\circ}$.

The value of $T_{0}$ was analysed by Cooper \& Gillespie (1952) and Cooper \& Linton (1952), and includes the time taken for the inoculum to reach a critical population by cell division, together with the lag period of growth. Since the generation time and lag period were determined for the 'Mayo' staphylococcus 
by broth experiments (see Table 1), it was possible to show this value on Figs. $1 a$ and $1 b$. By erecting a vertical line, from the point of intersection with the lag period on the time axis in Fig. $1 a$ to the inoculum size axis, the critical population is shown as $\log _{2} N_{0}=25 \cdot 2\left(N_{0}=39 \cdot 9 \times 10^{6}\right.$ organisms $/ \mathrm{ml}$. $)$. This value of $N_{0}$ we have called $N^{\prime}$.

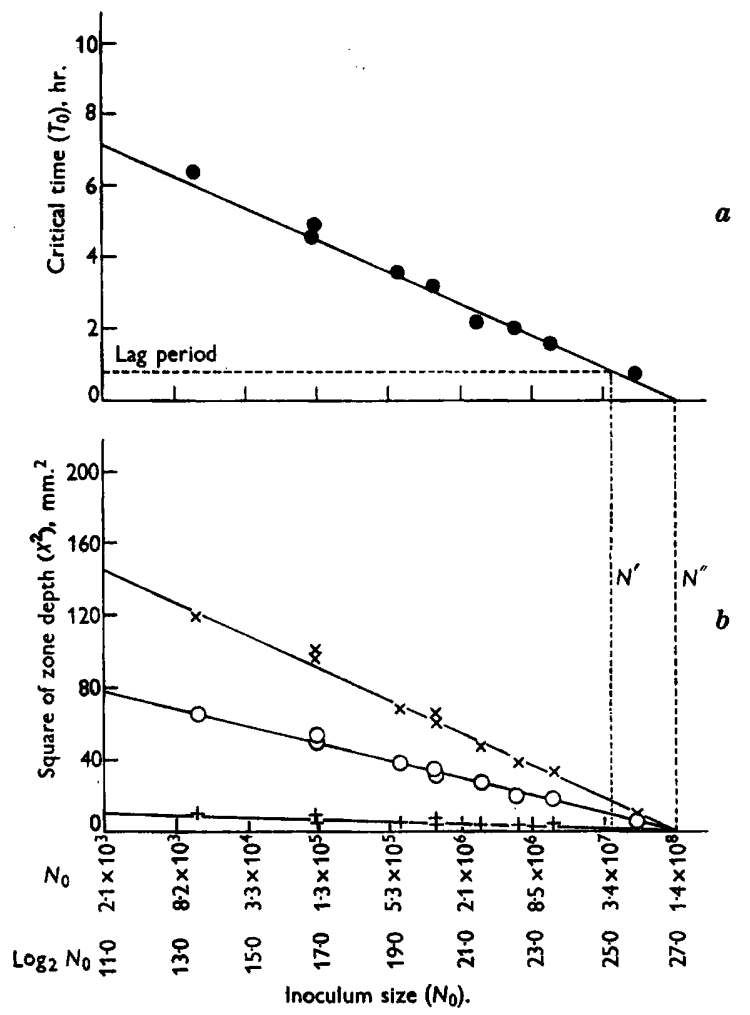

Fig. 3. The effect of inoculum size on critical time and the depth of inhibition zones produced by the action of streptomycin on staphylococcus, resistant strain, 6474 , at $35^{\circ}$. As in graph 1 ( $a$ and $b$ ). This strain has approximately the same growth rate, over the temperature range $27-40^{\circ}$ (see Tables 1 and 2), but a higher resistance to streptomycin $\left(m^{\prime}=7 \cdot 08 \mu \mathrm{g} . / \mathrm{ml}\right.$.) which was unaffected by temperature.

The straight-line graphs in Fig. $1 b$ have been calculated by substituting the experimental values of $T_{0}, m^{\prime}, m_{0}, D, N_{0}$ and $N^{\prime}$ in the formulae proposed by Cooper (1955). These are

$$
X^{2}=4 D\left(\log _{e} m_{0} / m^{\prime}\right) T_{0},
$$

and

$$
X^{2}=4 D\left(\log _{e} m_{0} / m^{\prime}\right)\left(L+G \log _{2} N^{\prime} / N_{0}\right) .
$$

From formula (1) it is seen that

$$
T_{0}=X^{2} / 4 D \log _{e}\left(m_{0} / m^{\prime}\right),
$$

and this is derived from the laws of diffusion and the conception of critical concentration at this time. From formulae (1) and (2) it is seen that

$$
T_{0}=L+G \log _{2}\left(N^{\prime} / N_{0}\right)
$$


and this is derived from the laws of growth of the organism, and gives the time required to achieve a critical population, $N^{\prime}$.

The agreement between the experimental values of $X^{2}$ and the calculated graphs is excellent.

\section{The influence of temperature}

In addition to the influence of inoculum size on inhibition zones, the effect of temperature was next investigated. Experiments were conducted at $27^{\circ}$, $30^{\circ}$, and $40^{\circ}$ for comparison with those at $35^{\circ}$, with the same test organism. Three concentrations of streptomycin were used $(10,100,1000 \mu \mathrm{g} . / \mathrm{ml}$.). Fig. $2 a$ shows the experimentally determined values of $T_{0}$ for each temperature, plotted against the logarithm of the inoculum size. The best straight lines through these points intersect the inoculum size axis at $\log _{2} N_{0}=27 \cdot 0$. In Fig. $2 b$ the experimental value of $X^{2}$ for each temperature with one concentration of streptomycin $(1000 \mu \mathrm{g} . / \mathrm{ml}$.) is plotted against the inoculum size. Fig. $2 a$ and $b$ show that whilst the slopes of each series of graphs vary with temperature, the $N^{\prime \prime}$ value remains constant. By comparing the lag periods experimentally determined in broth cultures, with those calculated and used in Figs. $2 a$ and $2 b$, it is shown that very good agreement was obtained (Table 1). The critical population $\left(N^{\prime}\right)$ is thus also constant and independent of temperature. This is shown more clearly by the enlarged inset graph.

Table 1. Streptomycin and a sensitive staphylococcus

The value of each variable determined experimentally and used for the theoretical calculations of graphs $X^{2} / \log _{2} N_{0}$ with streptomycin and sensitive strain of Staphylococcus aureus (Mayo 6473).

\begin{tabular}{|c|c|c|c|c|c|c|c|}
\hline \multirow[b]{2}{*}{ Temp. } & \multirow{2}{*}{$\begin{array}{l}\text { Viscosity* } \\
10^{2} \text { c.G.s. }\end{array}$} & \multirow{2}{*}{$\begin{array}{c}\text { Diffusion } \\
\text { coefficient } \\
D \mathrm{~mm} .^{2} / \mathrm{hr} \text {. }\end{array}$} & \multirow{2}{*}{$\begin{array}{c}\text { Critical } \\
\text { concen- } \\
\text { tration } \dagger \\
m^{\prime} \mu \mathrm{g} . / \mathrm{ml} . \\
\text { (average) }\end{array}$} & \multicolumn{2}{|c|}{$\begin{array}{l}\text { Zone-size constants } \\
\text { in agar }\end{array}$} & \multicolumn{2}{|c|}{$\begin{array}{l}\text { Growth-curve constants } \\
\text { in broth }\end{array}$} \\
\hline & & & & $L(h r.) \ddagger$ & $G(h r)$. & $L$ (hr.) & $G(\mathrm{hr})$. \\
\hline 27 & 0.855 & $0 \cdot 878$ & $\mathbf{2 \cdot 5}$ & $\mathbf{1} \cdot \mathbf{6 5}$ & 0.91 & $1 \cdot 65$ & $\mathbf{1 \cdot 3 5}$ \\
\hline $\mathbf{3 0}$ & $0 \cdot 800$ & 0.948 & $2 \cdot 1$ & $1 \cdot 13$ & 0.625 & $1 \cdot 125$ & $0 \cdot 75$ \\
\hline 35 & $0 \cdot 728$ & 1.06 & $1 \cdot 8$ & $0 \cdot 81$ & 0.45 & 0.825 & 0.575 \\
\hline \multirow[t]{2}{*}{40} & $0 \cdot 657$ & $1 \cdot 19$ & $2 \cdot 5$ & $0 \cdot 60$ & 0.33 & $0 \cdot 60$ & 0.42 \\
\hline & & & & \multicolumn{4}{|c|}{$L / G$ Av. ratio $1.81 \ddagger$} \\
\hline
\end{tabular}

Theoretical value used for the graphs:

$$
\begin{aligned}
\log _{2} N^{\prime} & =25 \cdot 2 & \log _{2} N^{\prime \prime} & =27 \cdot 0 \\
N^{\prime} & =38.9 \times 10^{6} & N^{\prime \prime} & =135.5 \times 10^{6}
\end{aligned}
$$

* Values quoted by Kaye \& Laby (1944).

$\dagger$ The deviations in $m^{\prime}$ with temperature were not experimentally significant. The variations recorded here are due to varied sensitivity on repeated subculture-but analogous variations occurred at $3^{\circ}$ as at other temperatures with the same strain. The average values in the table were used for theoretical graphs.

¥ The average ratio of $L$ (in broth) to $G$ (in agar) over the whole temperature range was used to calculate these $L$ values in agar.

The values of the constants used to calculate the graphs in Fig. $2 b$ are given in Table 1. The results reported in this paper have been obtained over a period of about two years, and it was found necessary to check repeatedly the sensitivity of the test strain to streptomycin. This was done by control determination of $m^{\prime}$ at $35^{\circ}$. When the $m^{\prime}$ value was found to have altered after 
repeated subculture, a freeze-dried ampoule of the strain was opened for further tests. The slight variations in the $m^{\prime}$ value of the test strain in Table 1 were due to an increased resistance found after certain subcultures before the freeze-drying technique was introduced.

\section{Table 2. Streptomycin and a resistant staphylococcus}

The values of each variable determined experimentally and used for the theoretical calculations of graphs $X^{2} / \log _{2} N_{0}$ with streptomycin and resistant strain of Staphylococcus aureus (Mayo 6474).

\begin{tabular}{|c|c|c|c|c|c|c|c|}
\hline \multirow{2}{*}{$\begin{array}{c}\text { Temp. } \\
\left({ }^{\circ} \mathrm{C} .\right)\end{array}$} & \multirow{2}{*}{$\begin{array}{l}\text { Viscosity* } \\
10^{2} \text { c.G.S. }\end{array}$} & \multirow{2}{*}{$\begin{array}{c}\text { Diffusion } \\
\text { coefficient } \\
D \mathrm{~mm} .^{2} / \mathrm{hr} .\end{array}$} & \multirow{2}{*}{$\begin{array}{c}\text { Critical } \\
\text { concen- } \\
\text { tration } \dagger \\
m^{\prime} \mu \mathrm{g} . / \mathrm{ml} \text {. } \\
\text { (average) }\end{array}$} & \multicolumn{2}{|c|}{$\begin{array}{l}\text { Zone-size constants } \\
\text { in agar }\end{array}$} & \multicolumn{2}{|c|}{$\begin{array}{c}\text { Growth-curve constant } \\
\text { in broth }\end{array}$} \\
\hline & & & & $L(\mathrm{hr}.) \ddagger$ & $G(\mathbf{h r})$. & $L$ (hr.) & $\boldsymbol{G}(\mathbf{h r})$. \\
\hline 27 & 0.855 & 0.878 & $7 \cdot 08$ & $1 \cdot 65$ & 0.91 & $1 \cdot 65$ & $1 \cdot 35$ \\
\hline 30 & 0.800 & 0.948 & $7 \cdot 08$ & $1 \cdot 13$ & 0.625 & $1 \cdot 125$ & 0.77 \\
\hline 35 & $0 \cdot 728$ & 1.06 & $7 \cdot 08$ & 0.825 & 0.45 & 0.825 & 0.575 \\
\hline \multirow[t]{2}{*}{40} & $0 \cdot 657$ & $1 \cdot 19$ & $7 \cdot 08$ & 0.60 & 0.33 & 0.60 & $0 \cdot 45$ \\
\hline & & & & \multicolumn{4}{|c|}{$L / G$ Av, ratio $1.81 \ddagger$} \\
\hline
\end{tabular}

Theoretical values used for the graphs:

$$
\begin{aligned}
\log _{2} N^{\prime} & =25 \cdot 2 & \log _{2} N^{\prime \prime} & =27 \cdot 0 \\
N^{\prime} & =38.9 \times 10^{6} & N^{\prime \prime} & =135.5 \times 10^{6}
\end{aligned}
$$

* Values quoted by Kaye \& Laby (1944).

$\dagger$ The strain remained quite consistent in its $m^{\prime}$ values throughout these series of experiments. Whenever a change was noticed, a new ampoule from the freeze-dried stock was obtained.

¥ The average ratio of $L$ (in broth) to $G$ (in agar) over the whole temperature range was used to calculate these $L$ values in agar.

\section{Experiments with the resistant mutant}

The effect of inoculum size at different temperatures was determined with the resistant staphylococcus mutant. This strain had the same growth rate as the sensitive parent strain but an increased $m^{\prime}$ value equal to $7 \cdot 08 \mu \mathrm{g}$. streptomycin $/ \mathrm{ml}$. The experimental results were plotted in the same manner as described for the sensitive strain. Figure 3 shows the results at $\mathbf{3 5}^{\circ}$, and it will be seen that, whilst the $X^{2}$ values are markedly reduced, the $N^{\prime}$ and $N^{\prime \prime}$ values are the same as for the sensitive staphylococcus. The analogous results obtained at other temperatures are summarized in Table 2. The use of a freezedried standard enabled the value of $m^{\prime}$ to be kept constant for all experiments.

\section{Generation time}

The slope of the graphs in Figs. $1 a, 2 a$ and $3 a$ are dependent upon the generation time $(G)$ of the test organism and therefore given a direct reading of this constant. These slopes were calculated from regression lines for the experimental points between $\log _{2} N_{0}=15 \cdot 0$ and $22 \cdot 0$, because zone edges in this range can be most accurately determined (see Table 1). A comparison between these values in solid media compared with determinations in broth shows the value for $G$ in agar was only $80 \%$ approximately of that obtained by broth experiments. Possible reasons for this are suggested later. 


\section{DISCUSSION}

The significance of critical time $T_{0}$

Cooper \& Woodman (1946) and Cooper \& Gillespie (1952) showed that preincubation of cultures decreased zone size. When the time interval between beginning incubation and adding the antibiotic $(h)$ became equal to $\boldsymbol{T}_{\mathbf{0}}$ the zone size diminished to zero, no matter what the concentration of antibiotic used $\left(m_{0}\right)$. With a standard size of inoculum, $T_{0}$ varied according to the temperature of incubation in such a way that the same number of generations resulted, a constant population $\left(N^{\prime}\right)$ being obtained. It is now shown that no matter what size of inoculum $\left(N_{0}\right)$ is used at time 0 , the length of $T_{0}$ is varied, so that this same critical population $\left(N^{\prime}\right)$ is achieved. At time $T_{0}$ the critical concentration $m^{\prime}$ fails for the first time to inhibit further growth.

When the graphs plotting $T_{0}$ against $\log _{2} N_{0}$ (Figs. $1 a, 2 a$ and $3 a$ ) are examined it will be seen that $T_{0}$ diminishes with increasing inoculum size. The experimental points agree well with formula (4):

$$
T_{0}=L+G \log _{2}\left(N^{\prime} / N_{0}\right)
$$

When $N_{0}=N^{\prime}$, then $T_{0}=L$, since $\log N^{\prime} / N_{0}=0$. With values of $N_{0}>N^{\prime}$, the value of $T_{0}$ becomes less than $L$, and the experimental points agree with the prolongation of the straight line until it meets the axis at $N^{\prime \prime}$ when $T_{0}=0$. This holds true for all values of $h$, including $h=0$.

Equation 4 may be written

$$
T_{0} / G=L / G+\log _{2}\left(N^{\prime} / N_{0}\right)
$$

and when $N_{0}=N^{\prime \prime}$ then $T_{0}=0$ and $0=L / G+\log N^{\prime} / N^{\prime \prime}$

$$
L / G=\log N^{\prime \prime}-\log N^{\prime}
$$

Eliminating $N^{\prime}$ from equations $(4 a)$ and (5)

$$
T_{0}=G \log N^{\prime \prime} / N_{0} \text {. }
$$

With an inoculum size of $N^{\prime \prime}$, the cocci are able to remove streptomycin to such an extent that the inhibitory concentration is not reached and growth occurs, giving no inhibition zones. With an inoculum of $N^{\prime}$ cocci we get inhibition zones. Their size shows they are formed within the lag period $(L)$. Cocci to the number of $N^{\prime}$, at the end of the lag period, combine with, and are inhibited by, the same amount of antibiotic as the larger number of cocci $N^{\prime \prime}$ at time 0 . The cocci showed no difference in sensitivity $\left(m^{\prime}\right)$ whatever the size of the inoculum, including populations with $N_{0}$ falling between $N^{\prime}$ and $N^{\prime \prime}$. It follows, therefore, that from time 0 to time $L$, the combining power of the cocci for streptomycin is increased, and as the experimental data agree with a straight-line graph up to $N_{0}=N^{\prime \prime}$ it seems certain that the full metabolic rate governing the synthesis of streoptomycin-absorbing sites has been achieved at time 0 in our experiments. It is known that under ideal conditions metabolic lag may be abolished, even though cell division lag remains (Gale, 1943). 
We find experimentally, for the staphylococcus used, that both $N^{\prime}$ and $N^{\prime \prime}$ prove to be constant even at different temperatures (Fig. 2). Since from equation (5) $\log N^{\prime \prime}-\log N^{\prime}=L / G$ this could only be so if $L / G$ was constant for all temperatures. Our experimental determinations of $L$ and $G$ in broth did give an approximate constant ratio up to $40^{\circ} \mathrm{C}$. The relative values of $L$ agreed with the $T_{0}$ values for $N_{0}=N^{\prime}$ at all temperatures. (See Tables 1 and 2.)

Table 2 shows that analogous results were obtained with a resistant mutant, chosen because it had the same growth rate as its prototype. The values of all constants in the formula remain the same as in Table 1, except the value of $m^{\prime}$. The value of $T_{0}$ is entirely determined by the time required for the population to reach $N^{\prime}$ per ml. and, as the theory predicts, is quite independent of $m^{\prime}$.

\section{The generation time $G$}

Johnson \& Lewin (1946) showed in the case of Escherichia coli that the variation of generation time with temperature was made up of two parts:

(a) Over the lower temperature range, growth occurred as though limited by the synthesis of one enzyme system, and the rate increased with temperature as would be expected from the thermodynamic constants involved.

(b) At higher temperatures, enzyme denaturation competed with enzyme synthesis and eventually lessened growth with increasing temperature. Heat denaturation when prolonged or excessive became irreversible; it may, however, be reversible but be more difficult to detect over short periods.

Apparently enzyme denaturation in our experiments is of little importance during the period of lag and the first few generations. Enzyme synthesis is thus the important consideration governing the formation of streptomycinabsorbing sites concerned in the formation of inhibition zones. Johnson \& Lewin (1946) showed that by plotting rate of growth (as percentage of maximum rate) against the reciprocal of the absolute temperature, a falling straight line resulted at low temperatures, a rising one at high temperatures, whilst middle temperature ranges were the resultant of the two.

When our values of $\boldsymbol{G}$ determined from zone size were plotted in this way, the experimental values fell on a straight line. This would be expected if streptomycin combined with protein before denaturation or if no protein denaturation occurred. Evidently the full rate of protein synthesis was established by time 0 , and the lag period in cell division was proportional to this rate, so that $L / G$ was constant. Values of $G$ may be calculated from antibiotic zones with considerable accuracy especially if $T_{0}$ is determined for light inocula. However, the value of $G$ calculated from the slope of the zonesize graphs was only $80 \%$ of the generation time determined from viable counts on broth cultures. A number of alternative explanations are possible; the true reason of this difference has not yet been determined. Most probably the greater size of the cocci during the early hours of growth is the cause (see Hershey, 1938, 1939). The absorption of toxic substances by agar might however contribute. 


\section{The significance of critical populations $N^{\prime}$ and $N^{\prime \prime}$}

The absorption of antibiotics by bacteria has been investigated by many workers. Berkman, Henry, Housewright, \& Henry (1948) showed that absorption of streptomycin by staphylococci reaches equilibrium rapidly, and that streptomycin could be completely eluted with $\mathrm{NaCl}$ solution. The amount of streptomycin absorbed and recovered was identical with resistant and susceptible strains, though the concentrations necessary in the external medium to inhibit growth, might be as different as 1 and $1000 \mu \mathrm{g}$. streptomycin/ ml. Eagle, Levy \& Fleischman (1955) showed by using radioactive penicillin that when inhibitory or lethal effects were produced, different species of bacteria of various degrees of susceptibility quantitatively accumulated the same amount of penicillin per organism. Sensitive strains were able to absorb from low concentrations in the medium, resistant strains only absorbed the same amount from higher concentrations. The plots of their results resemble Langmuir absorption curves, theoretically obtained by saturating more and more receptors in or on the organism until all were saturated, inhibition of growth (multiplication) occurring before this point. Some of their strains of staphylococcus (e.g. strain VI) showed a capacity for much further absorption (with higher concentrations) above this point. Humphrey \& Lightbown (1952) found that the absorption of streptomycin by staphylococci follows the Freundlich type $\left(X=20 \cdot 4 C^{0.24}\right)$ over the range of concentration examined, though saturation is expected to be achieved at higher concentrations (see Clarke, 1933). The electrophoretic mobility of staphylococci with absorbed streptomycin (studied by McQuillen, 1951), showed agreement with the Langmuir isotherm. A certain minimum external concentration is necessary to saturate sufficient active sites in order to inhibit division and in the case of staphylococci and streptomycin is reached before saturation. The critical concentration $m^{\prime}$ in diffusion experiments, necessary to prevent further division, is some 2-3 times greater than the minimum inhibitory concentration which will prevent growth when incorporated in broth or agar using a small inoculum. This is because part of the antibiotic (that which causes inhibition) is removed by absorption on the organisms which, under diffusion conditions, are initially present in excess at the zone edge. The external concentration is thus lowered to the minimum inhibitory concentration. The value of $m^{\prime}$ thus represents the sum of the absorbed and free streptomycin. When incorporated in the medium from the start, antibiotic is in excess and the external concentration is less affected when antibiotic is removed by the organisms of a small inoculum. The critical concentration $m^{\prime}$ is only capable of saturating enough active sites to inhibit growth in a limited number of organisms, namely $\boldsymbol{N}^{\prime}$ organisms during logarithmic growth and $N^{\prime \prime}$ organisms at the moment of inoculation, i.e. organisms harvested from a culture which develops a lag period before regular cell division.

In agreement with the above considerations the values of $N^{\prime}$ and $N^{\prime \prime}$ have remained constant, even when the value of $m^{\prime}$ has been varied considerably by methods expected to require greater external concentrations to achieve 
the same concentration within the organisms. This has been done (1) by using a mutant variant of our staphylococcal strain, which had much less sensitivity to streptomycin but the same growth rate at each temperature (see Fig. 3 for results at $\left.35^{\circ}\right),(2)$ by incorporating $\mathrm{MgSO}_{4}$ in the medium. This salt is a competitive antagonist for streptomycin absorption and experimental values of $m^{\prime}$ for variations of $\mathrm{Mg}$ from 66 to 5000 p.p.m. allowed $m^{\prime}$ to be varied from 1.5 to $25.0 \mu \mathrm{g}$. streptomycin $/ \mathrm{ml}$. in our medium (Linton, 1954). Results are expressed in terms of $\mathrm{Mg}$ concentration, but the sulphate ion is also important in this competition. The value of $m^{\prime}$ has been increased by this method up to 16 times without affecting $T_{0}$, so presumably the values of $N^{\prime}$ and $N^{\prime \prime}$ were unchanged.

The significance of $N^{\prime}$ and $N^{\prime \prime}$ in diffusion experiments is therefore that these are the number of cocci/ml. (young and old respectively) which by absorption of streptomycin can be inhibited by $m^{\prime} \mu \mathrm{g}$. streptomycin $/ \mathrm{ml}$. because after absorption the external concentration falls to the minimum inhibitory concentration as measured by incorporation experiments.

\section{The significance of critical concentration $m^{\prime}$}

We have for reasons of simplicity assumed that the streptomycin arriving by diffusion at an organism acts instantaneously, and if the concentration $<m^{\prime}$ is without effect, if the concentration $>m^{\prime}$ inhibits further cell division; such an assumption is obviously a mathematical approximation. Absorption of antibiotic by cells takes time, though the time to reach equilibrium between absorbed streptomycin and a particular concentration in the external medium is short-less than $10 \mathrm{~min}$. according to Humphrey \& Lightbown (1952). This period is less than the shortest possible generation time, and we may therefore consider that the absorbed antibiotic will inhibit the next cell division when the external concentration is still high enough to be at the minimum inhibitory concentration as determined by incorporation experiments in broth or agar.

Let $a=$ amount of antibiotic removed from the medium by one coccus.

Then $N^{\prime \prime} a=$ amount of antibiotic removed from $1 \mathrm{ml}$. of medium by $N^{\prime \prime}$ cocci.

Assuming that the amount of antibiotic remaining in the medium which is in equilibrium with that in the cocci is the amount required to produce bacteriostasis in the early hours of growth and that this is the minimum inhibitory concentration $(C)$, then the critical concentration as determined by diffusion experiments is given by

$$
m^{\prime}=N^{\prime \prime} a+C \quad \text { or } \quad a=\frac{m^{\prime}-C}{N^{\prime \prime}} .
$$

Humphrey \& Lightbown (1952) determined the amount of streptomycin absorbed by $1 \mathrm{mg}$. dry wt. staphylococei in different concentrations of antibiotic as given by $20 \cdot 4 C^{0.24}$. The number of cocci in $1 \mathrm{mg}$. dry wt. $=3 \times 10^{9}$ (Burroughs Wellcome and Co., London, tables supplied with Brown's opacity tubes), so

$$
a=\frac{20.4}{3 \times 10^{9}} C^{0.24} \text { and } N^{\prime \prime}=\frac{3 \times 10^{9}\left(m^{\prime}-C\right)}{20 \cdot 4 C^{0.24}} \text {. }
$$




\section{Inoculum size and inhibition zones}

The average value for $m^{\prime}$ at $35^{\circ}$ was $1 \cdot 8 \mu \mathrm{g}$. streptomycin $/ \mathrm{ml}$. Estimates of minimum inhibitory concentration from growth curves, and by incorporation of streptomycin in agar media, with this strain gave $C=0.9 \mu \mathrm{g}$. streptomcyin/ ml., approximately.

This gives

$$
N^{\prime \prime}=\frac{3 \times 10^{9}(1 \cdot 8-0 \cdot 9)}{20 \cdot 4(0 \cdot 9)^{0 \cdot 24}}=\frac{3 \times 10^{9}}{20 \cdot 4} 0^{0 \cdot 9^{0.76}}=1 \cdot 36 \times 10^{8}
$$

and

$$
\log _{2} N^{\prime \prime}=27 \cdot 00 \text {. }
$$

This value is identical with our results from the effects of inoculum size.

The use of Humphrey \& Lightbown's (1952) adsorption formula is justified for concentrations from 0 to 100 units, because although McQuillen (1951) by electrophoresis experiments showed that streptomycin adsorption on the surface of staphylococci fitted a Langmuir adsorption isotherm, saturation was not reached until over 1000 units were absorbed, and the part of the curve from 5 to $70 \%$ saturation was fitted accurately by the Freundlich straight line (see Clarke, 1933).

It can thus be seen that the critical concentration of streptomycin $m^{\prime}$ diffusing within the inhibition zone, is more than adequate to supply antibiotic to the organisms and stop further multiplication if they do not exceed $N^{\prime \prime}$ in number. Before the time $\boldsymbol{T}_{0}$ the number of cocci is insufficient to absorb enough antibiotic to lower the external concentration $m^{\prime}$ below the minimum inhibitory concentration (in this case $m^{\prime} / 2$ ), and cocci in equilibrium with such concentrations are inhibited. When more than $N^{\prime \prime}$ cocci are inoculated, or if by time $T_{0}$ they multiply to exceed $N^{\prime}$ in number, then the concentration of streptomycin in the external medium is lowered below the minimum inhibitory concentration. During the lag period an inoculum of $N^{\prime}$ cocci evidently develops as much absorbing capacity as $N^{\prime \prime}$ cocci at the moment of inoculation. No matter what the size of inoculum $N_{0}$, therefore, the critical time $T_{0}$ (when inhibition ceases) is determined by the time required for the culture to achieve a population density of $N^{\prime}$ cocci $/ \mathrm{ml}$. When the critical population ( $N^{\prime}$ during logarithmic growth, $N^{\prime \prime}$ for non-dividing cocci) is exceeded, absorption lowers the value of free streptomycin below the minimum inhibitory concentration. Further growth can then occur and absorption can deal with many times the concentration $m^{\prime}$ of streptomycin because the cocci are not saturated until a concentration over $1000 \mu \mathrm{g} . / \mathrm{ml}$. is present in the external medium.

\section{The lag period $L$}

The value of $L$ in the formula is the period from time 0 to the extrapolated point of onset of logarithmic growth. Provided active synthesis of antibiotic-absorbing sites is already fully established at time 0 , the value of $L$ may be incorporated with $N^{\prime}$ as a new population $N^{\prime \prime}$, containing the same number of absorbing sites at time 0 as $N^{\prime}$ population has at time $L$. This is only possible of course when $L / G$ is constant. When a lag in metabolism occurs after time 0 , then $L$ must be included for, like $h$ (pre-incubation time), it must be taken into account in determining diffusion time. It will be of opposite 
sign to $h$, because it is a time of pre-diffusion. But as Gale (1943) pointed out, increase in cell-nitrogen may show a steady increase from time of inoculation when the viable count gives a typical growth curve showing a lag phase. In this paper we have achieved such conditions and it is thus possible to eliminate $L$ completely by using $\log N^{\prime \prime}$ instead of $L / G+\log N^{\prime}$. Nevertheless, it is important to remember, when inoculum size is determined by viable count and not by mg. dry weight, that for values $<N^{\prime}$, allowance for increase in streptomycin utilization during the lag period is best made by including $L$ in the formula. The time when cell division begins is easily determined from growth curves.

The determination of $L$, at different temperatures, allows $N^{\prime}$ to be accurately fixed in relation to $N^{\prime \prime}$ and $G$ (see Fig. $2 a$ ). We have used determinations made with an inoculum size of $10^{6}$ organisms $/ \mathrm{ml}$. Hinshelwood (1946) discussed under what conditions the lag period might be expected to change with inoculum size. The conditions under which we prepared our dilutions for inoculation, however, were such as would be expected to minimize any such effect. They were: $(a)$ use of cocci of a constant age; $(b)$ considerable dilution in all cases to abolish the effect of accumulated toxic substances; $(c)$ the use of a highly nutritious medium to make the cocci independent of the need to accumulate diffusible intermediate compounds in biosynthesis.

\section{Experimental deviations from the theory}

If the explanation previously given of the difference between the value of $G$ used in the formulae and that determined from growth curves be accepted, then no unexpected deviations from theory have been observed. We conclude, therefore, that under the conditions of our experiments our original assumptions have been sufficiently closely approached, and that the experimental observations prove the essential truth of the theory, at least for the case of the action of streptomycin on our straphylococci.

Previous work suggests that the theory is capable of accurate application to other organisms and other antibiotics, provided that the conditions of the experiments are carefully controlled to fulfil the initial assumptions made, and that no destruction of antibiotic nor lysis of organisms occurs. With suitable modifications, it may be made to apply to other conditions. For example, the formula of Humphrey \& Lightbown (1952) or that of Vesterdal (1947) taking into account the falling concentration of antibiotic from a bead or small cup with a plate culture, may be used for the diffusion part of the formulae and combined with the growth curve part of our formula.

\section{Diffusion and population}

In solid media and gel-like structures such as body tissues, not only antibiotics but other toxic substances may spread by diffusion, therefore limiting growth in areas near their origin whilst being ineffective further away. The converse in which promotion of growth by diffusing nutrients occurs is, in many instances, also a means of controlling pupulations. The number of 
organisms in bacterial colonies has been shown to be a function of the distance between the colonies (Mayr-Harting, 1947), and the interchange of toxins and nutrients by diffusion necessitates relationships in time and populations. A proper understanding of the significance of critical concentration, time and population seems to us to be fundamental therefore for understanding growth and antibiotic action. The action of antibiotics in solid tissues is obviously limited by their diffusibility, but it has not, it seems to us, been realized that it is also limited by the critical population of organisms. If this be exceeded, i.e. if diffusion necessitates a critical time during which action must be achieved, then after this a thousandfold increase of antibiotic will be unable to stop further bacterial multiplication at such a site. The essential requirement of early therapy is thus shown to be a theoretical requirement as well as a clinical observation-and this is quite apart from such considerations as the development of adaptive enzymes or resistant mutants, or the destruction of antibiotic by bacterial substances.

(1) The formula

\section{Conclusions}

$$
\begin{aligned}
T_{0}-h & =L-h+G \log _{2} N^{\prime} / N_{0} & & \text { (growth) } \\
& =-h+G \log _{2} N^{\prime \prime} / N_{0} & & \text { (metabolism) } \\
& =X^{2} / 4 D \log _{e} m_{0} / m^{\prime} & & \text { (diffusion) }
\end{aligned}
$$

gives the relationship between the critical time $\left(T_{0}\right)$ and the size of the inhibition zone $(X)$ in terms of the concentration of antibiotic $(m)$, the rate of growth of the organism $(1 / G)$ and the number of organisms $(N)$. Lag period of cell division $=L$, and time of pre-incubation, before adding antibiotic $=h$.

(2) The critical concentration of diffusing antibiotic $\left(\mathrm{m}^{\prime}\right)$ at the zone edge at time $T_{0}$ is partly absorbed by the cell population. The amount remaining in solution is identical with the minimum inhibitory concentration determined by inoculating a few cocci into a large volume of medium incorporating antibiotic, or by determining the bacteriostatic concentration during the early hours of growth from growth curves.

(3) When the critical concentration $\left(m^{\prime}\right)$ is varied $(a)$ by the presence of substances competing for streptomycin adsorption centres, or $(b)$ by using resistant strains of staphylococci, the critical population is constant. This is probably because the amount absorbed/unit weight of cocci, necessary to inhibit multiplication, is constant.

(4) The critical time $\left(T_{0}\right)$ is the time required for the multiplying cells to achieve the critical population $\left(N^{\prime}\right)$.

(5) The critical population for non-dividing (but metabolizing) organisms $\left(N^{\prime \prime}\right)$ is greater than for multiplying ones $\left(N^{\prime}\right)$. Even during the lag period streptomycin-absorbing sites are being synthesized. If the inoculum size $\left(N_{0}\right)$ is greater than $N^{\prime}$, then the critical time $T_{0}$ is reduced below $L$. Inhibition zones are still formed unless $N_{0}>N^{\prime \prime}$, when $T_{0}$ and $X$ become nil.

(6) At different temperatures, though $G$ varies the critical population is constant. The latter is independent of inoculum size $\left(N_{0}\right)$ or antibiotic concentration $\left(m_{0}\right)$. 
(7) Critical population sets a limit to effective antibiotic therapy when the antibiotic has to reach the infected site by diffusion. Early therapy is essential for success, when the bacterial population is not being controlled by other defences.

We wish to acknowledge our gratitude to the Council of Scientific and Industrial Research, Government of India, for the appointment of one of us (S. N. Sehgal) as Assam Oil Company Scholar, to Miss B. Waters for the preparation of media, and to Dr G. Herdan for calculating the standard deviations of regression lines.

\section{REFERENCES}

Berkman, S., Henry, R. J., Housewright, R. D. \& Henry, J. (1948). Streptomycin. IV. Absorption of streptomycin by susceptible and resistant bacteria. Proc. Soc. exp. Biol., N.Y. 68, 65.

Burroughs, Wellcome and Co., London. Opacity tables A and B.

Clarke, A. J. (1933). The Mode of Action of Drugs on Cells, p. 44. London: Edward Arnold and Co.

Cooper, K. E. (1952). Note on the theory of inhibition zones. J. gen. Microbiol. 7, 13.

Cooper, K. E. (1955). Theory of antibiotic inhibition zones in agar media. Nature, Lond. 176, 510.

Cooper, K. E. \& Gillespie, W. A. (1952). The influence of temperature on Streptomycin inhibition zones in agar cultures. J. gen. Microbiol. 7, 1.

Cooper, K. E. \& Linton, A. H. (1952). The importance of the temperature during the early hours of incubation of agar plates in assays. J. gen. Microbiol. 7, 8.

Cooper, K. E. \& Woodman, D. (1946). The diffusion of antiseptics through agar gels, with special reference to the agar cup assay method of estimating the activity of penicillin. J. Path. Bact. 58, 75.

Eagle, H., Levvy, M. \& Fleischman, R. (1955). The binding of Penicillin in relation to its cytotoxic action. IV. The amounts bound by bacteria at ineffective, growth-inhibitory, bactericidal and maximally effective concentrations. J. Bact. 69, 167.

GaLe, E. F. (1943). Factors influencing the enzymic activities of Bacteria. Bact. Rev. 7, 139.

Gillissen, G. (1954). Der Agar-Diffusionstest (Lochtest) als Routineverfahren. Zbl. Bakt. (1 Org.), 161, 482.

Herdan, G. (1949). Assay of antiseptics using a Gaussian co-ordinate system. Research, 2, 590.

Herstey, A. D. (1938). Factors limiting bacterial growth. II. Growth without lag in Bacterium coli cultures. Proc. Soc. exp. Biol., N.Y. 38, 127.

HERSHEY, A. D. (1939). Factors limiting bacterial growth. IV. The age of the parent culture and the rate of growth of transplants of E. coli. J. Bact. 37, 285.

Hinshelwood, C. N. (1946). The Chemical Kinetics of the Bacterial Cell, p. 40. Oxford University Press.

Humphrey, J. H. \& LightBown, J. W. (1952). A general theory for plate assay of antibiotics with some practical applications. J. gen. Microbiol. 7, 129.

Ingram, G. I. C., Foxell, A. W. H. \& Armitage, P. (1953). The nature of the dose-response curve in penicillin plate assay. Antibiot. \& Chemother. 3, 1247.

Johnson, F. H. \& Lewin, I. (1946). The action of quinine on dehydrogenases of $\boldsymbol{E}$. coli. The disinfection of $\boldsymbol{E}$. coli in relation to temperature, hydrostatic pressure and quinine. J. cell. comp. Physiol. 28, 23, 47.

KAYE, C. W. C. \& LABy, T. H. (1944). Physical and Chemical Constants and some Mathematical Functions, 9th ed., p. 39. London: Longmans, Green and Co. 
Linton, A. H. (1949). Action of antibiotics on inhibition zones, Thesis M.Sc., Bristol University.

Linton, A. H. (1954). Studies in the viability of bacterial populations. Thesis Ph.D., Bristol University.

Linton, A. H. (1955). The relationship between growth rate of different bacteria and the formation of antibiotic inhibition zones. J. gen. Microbiol. 13, viii.

Masuyama, G. (1948). An assay method and a basic formula by use of one-dimensional diffusion. Kagaku, $17,158$.

Mayr-Harting, A. (1947). The growth of bacterial colonies and their viable population. J. Hyg., Camb. 45, 19.

McQuillen, K. (1951). The bacterial surface. IV. Effect of streptomycin on the electrophoretic mobility of Escherichia coli and Staphylococcus aureus. Biochem. brophys. Acta, 7, 54.

MiLes, A. A. \& Misra, S. S. (1938). The estimation of the bactericidal power of the blood. J. Hyg., Camb. 38, 732.

Mitchison, D. A. \& Sicer, C. C. (1949). A method of estimating streptomycin in serum and other body fluids by diffusion through Agar enclosed in glass tubes. J. gen. Microbiol. 3, 184.

Mryamura, S. (1953). Determination of the sensitivity of micro-organisms to antibiotics by the agar plate diffusion method. Antibiot. \& Chemother. 3, 903.

Schuesinger, F. G. (1954). Sensitivity tests by means of diffusion methods and their clinical interpretation. Acta med. scand. 148, 357.

SCHONFELD, J. K. (1953). The investigation of bacterial sensitivity in regard to antibiotics. Thesis, Leiden University.

Sykes, G., Lewis, D. G. \& Goshawk, B. (1951). Preliminary observations on the effect of some sugars on the assay of penicillin. Proc. Soc. appl. Bact. 14, 40.

Vesterdal, J. (1947). Studies on the inhibition zones observed in the agar cup method of penicillin assay. Acta path. microbiol. Scand. 24, 272.

(Received 9 December 1957) 